\title{
Differences in risk for SARS-CoV-2 infection among healthcare workers
}

\author{
K. Miriam Elfström ${ }^{\text {a }}$, Jonas Blomqvist ${ }^{\mathrm{a}}$, Peter Nilsson ${ }^{\mathrm{b}}$, Sophia Hober ${ }^{\mathrm{c}}$, Elisa Pin ${ }^{\mathrm{b}}$, \\ Anna Månberg $^{\mathrm{b}}$, Ville N. Pimenoff ${ }^{\mathrm{d}, \mathrm{e}}$, Laila Sara Arroyo Mühr ${ }^{\mathrm{d}}$, Kalle Conneryd Lundgren ${ }^{\mathrm{a}}$, \\ Joakim Dillner ${ }^{\text {a, }}$, \\ ${ }^{\text {a }}$ Karolinska University Hospital, Stockholm SE-141 86, Sweden \\ ${ }^{\mathrm{b}}$ Division of Affinity Proteomics, Department of Protein Science, KTH Royal Institute of Technology, SciLifeLab, Stockholm, Sweden \\ ${ }^{\mathrm{c}}$ Division of Protein Technology, Department of Protein Science, KTH Royal Institute of Technology, Stockholm, Sweden \\ ${ }^{\mathrm{d}}$ Karolinska Institute, Stockholm, Sweden \\ ${ }^{\mathrm{e}}$ University of Oulu, Oulu, Finland
}

\section{A R T I C L E I N F O}

Keywords:

SARS-CoV-2

COVID-19

Healthcare workers

\begin{abstract}
A B S T R A C T
Healthcare workers (HCWs) are a risk group for SARS-CoV-2 infection, but which healthcare work that conveys risk and to what extent such risk can be prevented is not clear. Starting on April 24th, 2020, all employees at work $(n=15,300)$ at the Karolinska University Hospital, Stockholm, Sweden were invited and 92\% consented to participate in a SARS-CoV-2 cohort study. Complete SARS-CoV-2 serology was available for $\mathrm{n}=12,928$ employees and seroprevalences were analyzed by age, sex, profession, patient contact, and hospital department. Relative risks were estimated to examine the association between type of hospital department as a proxy for different working environment exposure and risk for seropositivity, adjusting for age, sex, sampling week, and profession. Wards that were primarily responsible for COVID-19 patients were at increased risk (adjusted OR 1.95 (95\% CI 1.65-2.32) with the notable exception of the infectious diseases and intensive care units (adjusted OR 0.86 (95\% CI 0.66-1.13)), that were not at increased risk despite being highly exposed. Several units with similar types of work varied greatly in seroprevalences. Among the professions examined, nurse assistants had the highest risk (adjusted OR 1.62 (95\% CI 1.38-1.90)). Although healthcare workers, in particular nurse assistants, who attend to COVID-19 patients are a risk group for SARS-CoV-2 infection, several units caring for COVID-19 patients had no excess risk. Large variations in seroprevalences among similar units suggest that healthcare work-related risk of SARS-CoV-2 infection may be preventable.
\end{abstract}

\section{Introduction}

The first severe acute respiratory syndrome coronavirus 2 (SARSCoV-2) outbreak emerged in late 2019 in China (Zhang and Holmes, 2020), and quickly developed into a global coronavirus disease (COVID19) pandemic. Diagnostic surveillance of SARS-CoV-2 has reported more than 167 million SARS-CoV-2-infected and about 3.5 million COVID-19 deaths (2021-05-24). A major difficulty to prevent transmission of COVID-19 is that the pandemic is largely driven by asymptomatic and pre-symptomatic individuals, who are highly contagious (Bai et al., 2020; Cevik et al., 2021). Local, rapid COVID-19 outbreaks have been witnessed worldwide, particularly in elderly care homes (Burki, 2020; Feaster and Goh, 2020), nursing facilities (Arons et al., 2020) and among the healthcare workers (Meredith et al., 2020; Shah et al., 2019; Nguyen et al., 2020).

Nosocomial infections notoriously not only endanger the patient but jeopardize healthcare personnel, and consequently restrain the capacity of a healthcare unit. Indeed, accumulating evidence indicates that healthcare workers (HCWs) are highly exposed to SARS-CoV-2 infections, and that a large fraction of the infections stem from asymptomatic individuals (Moscola et al., 2020; Rudberg et al., 2020; Jespersen et al., 2020; Garcia-Basteiro et al., 2020). Hence, it is of utmost importance to quickly and efficiently detect and manage healthcare-related COVID-19 exposures.

To provide a greater understanding of SARS-CoV-2 infections in the hospital setting, we performed a very large-scale serosurvey that is unique in several respects: i) the study included HCWs at a major metropolitan hospital ii) the study had very high attendance iii) the data

\footnotetext{
* Corresponding author at: Karolinska University Laboratory, Karolinska University Hospital, Stockholm SE-14188, Sweden.

E-mail address: joakim.dillner@ki.se (J. Dillner).
} 


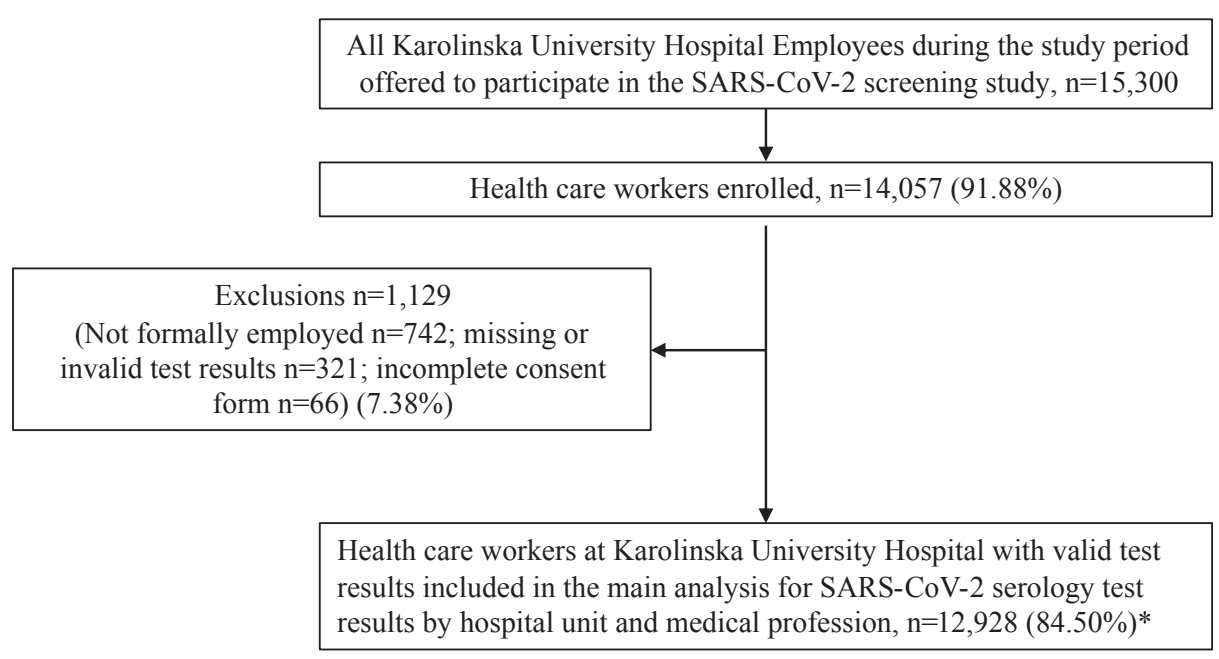

Fig. 1. STROBE flowchart of study participants.

used included specific individual-level information already recorded in the personnel administrative database on profession, information on whether the work involved patient contact, and continuous information on where the individual worked within the hospital; and iv) information recorded as to whether COVID-19 patients had been nursed in each unit or not. The study was performed very early in the first wave of the epidemic when routine testing was not available outside of the study. The risk of infection among the HCWs was estimated for each ward in the hospital and seroprevalences related to possible cofactors of infection, with particular focus on whether COVID-19 patients had been nursed at the wards.

\section{Methods}

\subsection{Study population and setting}

In response to the first wave of the COVID-19 pandemic, HCWs on duty at the Karolinska University Hospital (KUH) were invited to participate in a study that examined presence of antibodies to SARSCoV-2 in serum. KUH has about 15,300 employees in a variety of professions (physicians, nurses, nurse assistants, laboratory and administrative personnel as well as a variety of allied health professions) and is comprehensive in the types of health care provided. Healthy (i.e. asymptomatic) HCWs were recruited between April 23rd and June 24th, 2020. At that time, SARS-CoV-2 testing was not routinely available outside of the study (neither by serology nor PCR). All visits to the hospital by non-patients have been banned since March 13th, 2020. In Sweden, there was no policy mandating face masks or other PPE in health care settings. Public messaging stated that spread from asymptomatic subjects was very limited and that face masks did not largely prevent further spread of COVID-19. Citizens with symptoms that might be COVID-related were advised to stay at home. Although national guidelines were lax, many health care units may have taken individual management decisions on the use of PPE. During April 2020 there was risk for a general shortage of PPE and usage was likely somewhat affected by this. Participants signed a written informed consent that included permission to link to the hospital administrative databases for information on professional role (physician, nurse/midwife, nurse assistant, or other), sick leave records and hospital unit assignment (s).

The first COVID-19 patients were admitted to KUH in March 2020 and as the number of patients increased, hospital wards were repurposed and healthcare workers were re-assigned to care for the large influx of COVID-19 patients. The study participants were individually informed of their test results and positive results reported individually to the infectious disease authorities. The aggregated results were presented to the media in press conferences as well as presented to authorities and made available to all unit heads across hospital departments. The hospital managing director (KCL) was part of the study team and had continuous access to the data.

The study was approved by the National Ethical Review Agency of Sweden (Decision number 2020-01620). Trial registration number: ClinicalTrials.gov NCT04411576.

\subsection{Serological analyses}

Whole blood was prepared as serum by centrifugation in serumseparating tubes at $2000 \times \mathrm{g}$ for $10 \mathrm{~min}$. A heat treatment at $56 \mathrm{de}-$ grees $\mathrm{C}$ for $30 \mathrm{~min}$ was performed for virus inactivation and the samples were subsequently stored at -20 degrees $C$ until further analysis.

SARS-CoV-2 IgG antibodies were analysed using three different virus protein variants, namely the trimeric full-length Spike protein (Wrapp et al., 2020) expressed in HEK cells, the Spike S1 domain expressed in $\mathrm{CHO}$ cells and the Nucleocapsid protein expressed in E. coli. The serum samples were processed as previously described (Neiman et al., 2019) and analysed in a 384-plate format using a multiplex bead-based assay (Luminex corp.) with IgG detection towards all three viral antigens in parallel.

The performance of the serology assay was evaluated using 154 positive controls (defined as Covid-19 patients sampled at least 15 days after a positive PCR test) and 321 negative controls (defined as samples collected 2019 and earlier in the same region). The negative controls included 26 individuals with confirmed infections of other Coronaviruses than SARS-CoV-2. Based on these samples, the assay showed a $99.4 \%$ sensitivity and $99.1 \%$ specificity. Seropositivity was defined for each antigen as mean +6 SD of 12 standardized negative control samples included in each analysis plate. For a sample to be assigned as IgG positive, a sample was required to be positive against at least two of the three included viral antigens. Captured IgG antibodies were detected by fluorescent anti-hIgG (Invitrogen, H10104) and reported as relative fluorescence intensity (AU).

\subsection{Statistical analysis}

For this study on risk of SARS-CoV-2 infection by type of healthcare work, participating healthcare workers were categorized based on their profession (physician, nurse/midwife, nurse assistant, or other), whether or not they had any patient contact, and their place of employment within the hospital. Hospital units were then categorized as intensive care units (ICU), COVID wards (meaning non-ICU units that cared for COVID-19 patients at some point during the study period) and 
Table 1

Distribution of background covariates among 12,928 employees of the Karolinska University Hospital.

\begin{tabular}{|c|c|c|c|}
\hline & n (\%) & $\begin{array}{l}\text { Serology positive } \mathrm{n}(\%, 95 \% \\
\text { CI) }\end{array}$ & p-value \\
\hline \multicolumn{4}{|l|}{ Age } \\
\hline$\leq 29$ & $1522(11.8)$ & $249(16.4,14.6-18.3)$ & \multirow[t]{5}{*}{$<0.0001^{*}$} \\
\hline $30-39$ & $3172(24.5)$ & $382(12.0,11.0-13.2)$ & \\
\hline $40-49$ & $3238(25.1)$ & $370(11.4,10.4-12.6)$ & \\
\hline $50-59$ & $3066(23.7)$ & $313(10.2,9.2-11.3)$ & \\
\hline$\geq 60$ & $1930(14.9)$ & $166(8.6,7.4-9.9)$ & \\
\hline \multicolumn{4}{|l|}{ Sex } \\
\hline Female & $\begin{array}{l}10203 \\
(78.9)\end{array}$ & $1,170(11.5,10.9-12.1)$ & \multirow[t]{2}{*}{$0.8945^{* *}$} \\
\hline Male & $2,725(21.1)$ & $310(11.4,10.2-12.6)$ & \\
\hline \multicolumn{4}{|l|}{ Sampling week } \\
\hline Week 17-18 & $6801(52.6)$ & $699(10.3,9.6-11.0)$ & \multirow[t]{3}{*}{$<0.0001^{*}$} \\
\hline Week 19-20 & $4274(33.1)$ & $539(12.6,11.7-13.6)$ & \\
\hline Week 21+ & $1853(14.3)$ & $242(13.1,11.6-14.7)$ & \\
\hline \multicolumn{4}{|l|}{ Profession } \\
\hline Other personnel & $4043(31.3)$ & $385(9.5,8.7-10.5)$ & \multirow[t]{4}{*}{$<0.0001^{* *}$} \\
\hline Nurse/midwife & $4027(31.2)$ & $486(12.1,11.1-13.1)$ & \\
\hline Nurse assistant & $2532(19.6)$ & $389(15.4,14.0-16.8)$ & \\
\hline Physician & $2326(18.0)$ & $220(9.5,8.3-10.7)$ & \\
\hline \multicolumn{4}{|l|}{ Patient contact } \\
\hline No patient contact & $3671(28.4)$ & $356(9.7,8.8-10.7)$ & \multirow[t]{2}{*}{$<0.0001^{* *}$} \\
\hline Patient contact & $9257(71.6)$ & $1124(12.1,11.5-12.8)$ & \\
\hline \multicolumn{4}{|l|}{ Type of hospital unit } \\
\hline Other & $\begin{array}{l}11,259 \\
(87.1)\end{array}$ & $1192(10.6,10.0-11.2)$ & \multirow[t]{3}{*}{$<0.0001$} \\
\hline COVID-19 ward & $1031(8.0)$ & $225(21.8,19.4-24.5)$ & \\
\hline $\begin{array}{l}\text { Intensive care unit } \\
\text { (ICU) }\end{array}$ & $638(4.9)$ & $63(9.9,7.8-12.4)$ & \\
\hline
\end{tabular}

* Cochran Armitage Trend Test.

** Chi square test.

"other" (including research, administrative and management units, that did not have routine contact with COVID-19 patients).

Baseline data showing the distribution of HCW roles and places of employment within the hospital were estimated overall and by seroprevalence. Chi square tests and Cochran Armitage tests of trend were used to examine the association between covariates and seroprevalence. A multivariate logistic regression was run examining the association between type of hospital ward and serology positivity, adjusting for age, sex, sampling week, and professional role. Patient contact was not included in the model since it was strongly associated with the professional role of the HCWs. For the analysis of risk of SARS-CoV-2 infection associated with each hospital unit, HCWs with two or more affiliations listed were assigned to a ward-type using the following logic: HCWs were assumed to have contributed to COVID care, and then ICU or "other" units in that order given the need for additional support in the COVID wards. Hospital units with $<50$ participating HCWs were excluded from the unit-specific analysis, resulting in the removal of 44 units. The hospital units that cared for COVID-19 patients were noted and then serology positivity was reported as a prevalence with $95 \%$ confidence intervals. Using a case-cohort design, odds ratios were calculated comparing the odds of serology positivity in the unit to the odds of serology positivity in a reference group consisting of all 3671 employees registered as not having any patient contact in their work tasks.

The data were prepared and analyzed using SAS 9.4 Cary, NC, USA and a p-value of $<0.05$ was deemed significant.

\section{Results}

To assess risk of SARS-CoV-2 exposure by type of healthcare work, we enrolled 14,057 HCWs at work between April and June 2020 at
Table 2

Association between type of hospital ward and serology positivity, adjusted for co-factors.

\begin{tabular}{lll}
\hline & OR (95\% CI) Unadjusted & OR (95\% CI) Mutually adjusted \\
\hline Age & & \\
$\leq 29$ & 1.00 & 1.00 \\
$30-39$ & $0.70(0.59-0.83)$ & $0.78(0.65-0.93)$ \\
$40-49$ & $0.66(0.55-0.79)$ & $0.76(0.64-0.91)$ \\
$50-59$ & $0.58(0.49-0.70)$ & $0.67(0.56-0.80)$ \\
$\geq 60$ & $0.48(0.39-0.59)$ & $0.57(0.46-0.70)$ \\
Sex & & \\
Females & 1.00 & 1.00 \\
Males & $0.99(0.87-1.13)$ & $1.14(0.99-1.31)$ \\
Sampling week & & \\
Weeks 17-18 & 1.00 & 1.00 \\
Weeks 19-20 & $1.26(1.12-1.42)$ & $1.32(1.17-1.49)$ \\
Weeks 21+ & $1.31(1.12-1.53)$ & $1.45(1.24-1.71)$ \\
Profession & & \\
Other personnel & 1.00 & 1.00 \\
Nurse/midwife & $1.30(1.13-1.50)$ & $1.28(1.11-1.49)$ \\
Nurse assistant & $1.73(1.48-2.01)$ & $1.62(1.38-1.90)$ \\
Physician & $0.99(0.83-1.18)$ & $1.07(0.89-1.28)$ \\
Type of hospital unit & & \\
Other & 1.00 & 1.00 \\
COVID ward & $2.36(2.01-2.77)$ & $0.86(0.66-1.13)$ \\
ICU & $0.93(0.71-1.21)$ & \\
\hline
\end{tabular}

Karolinska University Hospital (92\% of all HCWs). We obtained valid serology results and formal employment status for 12,928 participants (Fig. 1). Significant differences in Covid-19 seropositivity were observed by age, sampling (calendar) week, professional role of HCWs, and type of hospital unit (Table 1). A trend of decreased SARS-CoV-2 infection with age from $16.4 \%$ (95\% CI 14.6-18.3) among HCWs under the age of 30 to $8.6 \%$ (95\% CI 7.4-9.9) among HCWs over the age of 60 was found (p-value for trend, <0.0001) (Table 1). Moreover, in the multivariate logistic regression model, a significant almost two-fold increased risk SARS-CoV-2 exposure was found for HCWs in hospital units caring for COVID-19 patients (adjusted OR 1.95 (95\% CI 1.65-2.32)) as compared to units not caring for COVID-19 patients. Notably, ICU wards were not at an increased risk as compared to other wards despite intensive work with COVID patients (adjusted OR 0.86 (95\% CI 0.66-1.13)). Differences in risk for seropositivity were also seen by profession and sampling week. Nurse assistants had the highest risk for seropositivity (adjusted OR 1.62 (95\% CI 1.38-1.90)), followed by nurses and midwives (adjusted OR 1.28 (95\% CI 1.11-1.49)) as compared to a reference group of other healthcare personnel. Physicians did not have a significantly increased risk. The highest risk for SARS-CoV-2 seropositivity was observed in the last weeks of sampling in the study (calendar weeks 21 + ) as compared to the first weeks (calendar weeks 17-18) (adjusted OR 1.45 (95\% CI 1.24-1.71)) (Table 2).

To comprehensively explore the risk of SARS-CoV-2 exposure for HCWs in different hospital settings, we compared the odds of seropositivity in each hospital unit to the odds of seropositivity in our reference group, which consisted of the hospital staff 1 without any patient contact in their work (Table 3). HCWs in six out of ten COVID wards had more than two-fold increased risk of SARS-CoV-2 exposure compared to HCWs without patient contact. The highest, almost three-fold increased risk for SARS-CoV-2 exposure was observed for HCWs in units that had been repurposed to nurse COVID-19 patients but that would typically care for patients with non-infectious diseases (e.g. neurology, geriatrics, and cardiology). There were 5/55 wards who had not nursed COVID-19 patients but still had a similar, nearly two-fold increased risk of SARSCoV-2 exposure. 
Table 3

Serology positivity by hospital unit, odds of serology positivity for each hospital unit compared to odds among participants with no patient contact.

\begin{tabular}{|c|c|c|c|}
\hline Hospital unit & $\begin{array}{l}\text { n serology } \\
\text { positive/total \# } \\
\text { part. in unit }\end{array}$ & $\%(95 \% \mathrm{CI})$ & $\begin{array}{l}\text { OR }(95 \% \mathrm{CI}) \\
\text { Unadjusted }\end{array}$ \\
\hline${ }^{\S}$ Pelvic cancer & $52 / 361$ & $\begin{array}{l}14.4 \\
(11.2-18.4)\end{array}$ & $\begin{array}{l}1.52 \\
(1.11-2.08)\end{array}$ \\
\hline Upper abdominal cancers & $23 / 198$ & $\begin{array}{l}11.6 \\
(7.9-16.8)\end{array}$ & $\begin{array}{l}1.18 \\
(0.76-1.86)\end{array}$ \\
\hline $\begin{array}{l}\text { Breast cancers, endocrine } \\
\text { tumors and sarcomas }\end{array}$ & $31 / 141$ & $\begin{array}{l}22.0 \\
(15.9-29.5)\end{array}$ & $\begin{array}{l}2.54 \\
(1.68-3.85)\end{array}$ \\
\hline ENT, hearing and balance & $23 / 231$ & $\begin{array}{l}10.0 \\
(6.7-14.5)\end{array}$ & $\begin{array}{l}1.00 \\
(0.64-1.56)\end{array}$ \\
\hline Cardiology, ward & $53 / 213$ & $\begin{array}{l}24.9 \\
(19.6-31.1)\end{array}$ & $\begin{array}{l}2.99 \\
(2.15-4.16)\end{array}$ \\
\hline Seriatrics, ward & $46 / 185$ & $\begin{array}{l}24.9 \\
(19.2-31.6)\end{array}$ & $\begin{array}{l}2.99 \\
(2.10-4.25)\end{array}$ \\
\hline $\begin{array}{l}{ }^{8} \text { Inflammation and } \\
\text { infectious diseases, ward }\end{array}$ & $45 / 343$ & $\begin{array}{l}13.1 \\
(10.0-17.1)\end{array}$ & $\begin{array}{l}1.36 \\
(0.98-1.90)\end{array}$ \\
\hline${ }^{5}$ Neurology, ward & $54 / 207$ & $\begin{array}{l}26.1 \\
(20.6-32.5)\end{array}$ & $\begin{array}{l}3.19 \\
(2.29-4.43)\end{array}$ \\
\hline${ }^{8}$ Emergency care, ward 1 & $50 / 249$ & $\begin{array}{l}20.1 \\
(15.6-25.5)\end{array}$ & $\begin{array}{l}2.27 \\
(1.63-3.16)\end{array}$ \\
\hline Emergency care, ward 2 & $82 / 405$ & $\begin{array}{l}20.3 \\
(16.6-24.4)\end{array}$ & $\begin{array}{l}2.29 \\
(1.75-3.00)\end{array}$ \\
\hline${ }^{8}$ ICU \& thorax surgery & $59 / 760$ & $7.8(6.1-9.9)$ & $\begin{array}{l}0.76 \\
(0.57-1.01)\end{array}$ \\
\hline${ }^{\S}$ Pediatric ICU & $38 / 317$ & $\begin{array}{l}12.0 \\
(8.9-16.0)\end{array}$ & $\begin{array}{l}1.23 \\
(0.86-1.76)\end{array}$ \\
\hline${ }^{\S}$ Neonatology & $35 / 367$ & $\begin{array}{l}9.5 \\
(6.9-13.0)\end{array}$ & $\begin{array}{l}0.95 \\
(0.66-1.37)\end{array}$ \\
\hline $\begin{array}{l}\text { R\&D management, } \\
\text { Division for research }\end{array}$ & $6 / 93$ & $\begin{array}{l}6.5 \\
(3.0-13.4)\end{array}$ & $\begin{array}{l}0.62 \\
(0.27-1.44)\end{array}$ \\
\hline $\begin{array}{l}\text { R\&D management, } \\
\text { Division for education }\end{array}$ & $6 / 86$ & $\begin{array}{l}7.0 \\
(3.2-14.4)\end{array}$ & $\begin{array}{l}0.68 \\
(0.29-1.56)\end{array}$ \\
\hline ICU, Resident physicians & $1 / 65$ & $1.5(0.3-8.2)$ & $\begin{array}{l}0.14 \\
(0.02-1.02)\end{array}$ \\
\hline $\begin{array}{l}\text { Development and } \\
\text { administration }\end{array}$ & $3 / 63$ & $\begin{array}{l}4.8 \\
(1.6-13.1)\end{array}$ & $\begin{array}{l}0.45 \\
(0.14-1.45)\end{array}$ \\
\hline Human resources & $6 / 101$ & $\begin{array}{l}5.9 \\
(2.8-12.4)\end{array}$ & $\begin{array}{l}0.57 \\
(0.25-1.31)\end{array}$ \\
\hline Staffing administration & $16 / 111$ & $\begin{array}{l}14.4 \\
(9.1-22.1)\end{array}$ & $\begin{array}{l}1.52 \\
(0.88-2.61)\end{array}$ \\
\hline Clinical nutrition & $7 / 50$ & $\begin{array}{l}14.0 \\
(7.0-27.2)\end{array}$ & $\begin{array}{l}1.47 \\
(0.66-3.29)\end{array}$ \\
\hline Social work & $5 / 85$ & $\begin{array}{l}5.9 \\
(2.5-13.0)\end{array}$ & $\begin{array}{l}0.56 \\
(0.23-1.40)\end{array}$ \\
\hline $\begin{array}{l}\text { Occupational therapy and } \\
\text { physiotherapy }\end{array}$ & $17 / 195$ & $\begin{array}{l}8.7 \\
(5.5-13.5)\end{array}$ & $\begin{array}{l}0.86 \\
(0.52-1.44)\end{array}$ \\
\hline Emergency care & $42 / 303$ & $\begin{array}{l}13.9 \\
(10.4-18.2)\end{array}$ & $\begin{array}{l}1.45 \\
(1.03-2.05)\end{array}$ \\
\hline Neuroradiology & $4 / 96$ & $\begin{array}{l}4.2 \\
(1.6-10.2)\end{array}$ & $\begin{array}{l}0.39 \\
(0.14-1.08)\end{array}$ \\
\hline $\begin{array}{l}\text { Medical radiation and } \\
\text { nuclear medicine }\end{array}$ & $11 / 119$ & $\begin{array}{l}9.2 \\
(5.2-15.8)\end{array}$ & $\begin{array}{l}0.92 \\
(0.49-1.73)\end{array}$ \\
\hline Clinical physiology & $7 / 69$ & $\begin{array}{l}10.1 \\
(5.0-19.5)\end{array}$ & $\begin{array}{l}1.02 \\
(0.46-2.25)\end{array}$ \\
\hline Radiology 1 & $17 / 166$ & $\begin{array}{l}10.2 \\
(6.5-15.8)\end{array}$ & $\begin{array}{l}1.03 \\
(0.62-1.72)\end{array}$ \\
\hline Radiology 2 & $9 / 96$ & $\begin{array}{l}9.4 \\
(5.0-16.9)\end{array}$ & $\begin{array}{l}0.93 \\
(0.47-1.87)\end{array}$ \\
\hline Perioperative medicine 1 & $40 / 396$ & $\begin{array}{l}10.1 \\
(7.5-13.5)\end{array}$ & $\begin{array}{l}1.01 \\
(0.72-1.43)\end{array}$ \\
\hline Perioperative medicine 2 & $25 / 148$ & $\begin{array}{l}16.9 \\
(11.7-23.8)\end{array}$ & $\begin{array}{l}1.84 \\
(1.18-2.86)\end{array}$ \\
\hline Newborn screening lab & $4 / 70$ & $\begin{array}{l}5.7 \\
(2.2-13.8)\end{array}$ & $\begin{array}{l}0.55 \\
(0.20-1.51)\end{array}$ \\
\hline Pathology and cytology & $40 / 400$ & $\begin{array}{l}10.0 \\
(7.4-13.3)\end{array}$ & $\begin{array}{l}1.00 \\
(0.71-1.42)\end{array}$ \\
\hline Clinical genetics & $5 / 79$ & $\begin{array}{l}6.3 \\
(2.7-14.0)\end{array}$ & $\begin{array}{l}0.61 \\
(0.24-1.52)\end{array}$ \\
\hline Clinical pharmacology & $6 / 137$ & $4.4(2.0-9.2)$ & $\begin{array}{l}0.41 \\
(0.18-0.94)\end{array}$ \\
\hline $\begin{array}{l}\text { Clinical immunology and } \\
\text { transfusion medicine }\end{array}$ & $36 / 292$ & $\begin{array}{l}12.3 \\
(9.0-16.6)\end{array}$ & $\begin{array}{l}1.11 \\
(0.77-1.60)\end{array}$ \\
\hline Clinical chemistry & $22 / 320$ & & \\
\hline
\end{tabular}

Table 3 (continued)

\begin{tabular}{|c|c|c|c|}
\hline Hospital unit & $\begin{array}{l}\text { n serology } \\
\text { positive/total \# } \\
\text { part. in unit }\end{array}$ & $\%(95 \% \mathrm{CI})$ & $\begin{array}{l}\text { OR }(95 \% \mathrm{CI}) \\
\text { Unadjusted }\end{array}$ \\
\hline & & $\begin{array}{l}6.9 \\
(4.6-10.2)\end{array}$ & $\begin{array}{l}0.67 \\
(0.43-1.04)\end{array}$ \\
\hline Clinical microbiology & $18 / 266$ & $\begin{array}{l}6.8 \\
(4.3-10.4)\end{array}$ & $\begin{array}{l}0.66 \\
(0.40-1.07)\end{array}$ \\
\hline $\begin{array}{l}\text { Point-of-care laboratory } \\
\text { network }\end{array}$ & $24 / 237$ & $\begin{array}{l}10.1 \\
(6.9-14.6)\end{array}$ & $\begin{array}{l}1.02 \\
(0.66-1.57)\end{array}$ \\
\hline Cardiology & $22 / 183$ & $\begin{array}{l}12.0 \\
(8.1-17.5)\end{array}$ & $\begin{array}{l}1.23 \\
(0.78-1.95)\end{array}$ \\
\hline Infectious diseases & $4 / 61$ & $\begin{array}{l}6.6 \\
(2.6-15.7)\end{array}$ & $\begin{array}{l}0.63 \\
(0.23-1.76)\end{array}$ \\
\hline $\begin{array}{l}\text { Gastric, skin. and } \\
\text { rheumatic disorders }\end{array}$ & $11 / 165$ & $\begin{array}{l}6.7 \\
(3.8-11.5)\end{array}$ & $\begin{array}{l}0.65 \\
(0.35-1.20)\end{array}$ \\
\hline Endocrinology & $5 / 83$ & $\begin{array}{l}6.0 \\
(2.6-13.3)\end{array}$ & $\begin{array}{l}0.58 \\
(0.23-1.44)\end{array}$ \\
\hline Nephrology & $9 / 159$ & $\begin{array}{l}5.7 \\
(3.0-10.4)\end{array}$ & $\begin{array}{l}0.54 \\
(0.27-1.07)\end{array}$ \\
\hline $\begin{array}{l}\text { Gynecology and } \\
\text { reproductive medicine }\end{array}$ & $17 / 95$ & $\begin{array}{l}17.9 \\
(11.5-26.8)\end{array}$ & $\begin{array}{l}1.97 \\
(1.15-3.37)\end{array}$ \\
\hline Neurology & $3 / 65$ & $\begin{array}{l}4.6 \\
(1.6-12.7)\end{array}$ & $\begin{array}{l}0.44 \\
(0.14-1.40)\end{array}$ \\
\hline Radiation & $5 / 86$ & $\begin{array}{l}5.8 \\
(2.5-12.9)\end{array}$ & $\begin{array}{l}0.56 \\
(0.22-1.39)\end{array}$ \\
\hline Hematology & $14 / 169$ & $\begin{array}{l}8.3 \\
(5.0-13.4)\end{array}$ & $\begin{array}{l}0.82 \\
(0.47-1.43)\end{array}$ \\
\hline $\begin{array}{l}\text { Head \& neck, lung and } \\
\text { skin cancers }\end{array}$ & $14 / 204$ & $\begin{array}{l}6.9 \\
(4.1-11.2)\end{array}$ & $\begin{array}{l}0.67 \\
(0.38-1.16)\end{array}$ \\
\hline Transplantation medicine & $6 / 75$ & $\begin{array}{l}8.0 \\
(3.7-16.4)\end{array}$ & $\begin{array}{l}0.79 \\
(0.34-1.82)\end{array}$ \\
\hline $\begin{array}{l}\text { Plastic surgery and } \\
\text { craniofacial diseases }\end{array}$ & $12 / 74$ & $\begin{array}{l}16.2 \\
(9.5-26.2)\end{array}$ & $\begin{array}{l}1.75 \\
(0.93-3.28)\end{array}$ \\
\hline $\begin{array}{l}\text { Pediatric surgery and } \\
\text { medicine, ward }\end{array}$ & $25 / 202$ & $\begin{array}{l}12.4 \\
(8.5-17.6)\end{array}$ & $\begin{array}{l}1.28 \\
(0.83-1.97)\end{array}$ \\
\hline $\begin{array}{l}\text { Pediatric emergency care, } \\
\text { ward }\end{array}$ & $24 / 185$ & $\begin{array}{l}13.0 \\
(8.9-18.6)\end{array}$ & $\begin{array}{l}1.35 \\
(0.86-2.10)\end{array}$ \\
\hline $\begin{array}{l}\text { Pregnancy and deliveries, } \\
\text { ward }\end{array}$ & $52 / 400$ & $\begin{array}{l}13.0 \\
(10.1-16.7)\end{array}$ & $\begin{array}{l}1.35 \\
(0.99-1.84)\end{array}$ \\
\hline $\begin{array}{l}\text { Pediatric surgery and } \\
\text { medicine, ward }\end{array}$ & $16 / 95$ & $\begin{array}{l}16.8 \\
(10.6-25.6)\end{array}$ & $\begin{array}{l}1.83 \\
(1.06-3.17)\end{array}$ \\
\hline $\begin{array}{l}\text { Pediatric orthopedics, } \\
\text { ward }\end{array}$ & $15 / 130$ & $\begin{array}{l}11.5 \\
(7.1-18.2)\end{array}$ & $\begin{array}{l}1.18 \\
(0.68-2.04)\end{array}$ \\
\hline Neurosurgery, ward & $8 / 66$ & $\begin{array}{l}12.1 \\
(6.3-22.1)\end{array}$ & $\begin{array}{l}1.25 \\
(0.59-2.63)\end{array}$ \\
\hline $\begin{array}{l}\text { Management, Imaging \& } \\
\text { Functional diagnostics } \\
\text { div. }\end{array}$ & $12 / 123$ & $\begin{array}{l}9.8 \\
(5.7-16.3)\end{array}$ & $\begin{array}{l}0.98 \\
(0.53-1.79)\end{array}$ \\
\hline $\begin{array}{l}\text { Management, Hospital } \\
\text { Laboratory div. }\end{array}$ & $12 / 71$ & $\begin{array}{l}16.9 \\
(9.9-27.3)\end{array}$ & $\begin{array}{l}1.84 \\
(0.98-3.45)\end{array}$ \\
\hline Management, ICU div. & $20 / 132$ & $\begin{array}{l}15.2 \\
(10.0-22.3)\end{array}$ & $\begin{array}{l}1.62 \\
(0.99-2.63)\end{array}$ \\
\hline $\begin{array}{l}\text { Management, Geriatrics } \\
\text { div. }\end{array}$ & $6 / 58$ & $\begin{array}{l}10.3 \\
(4.8-20.8)\end{array}$ & $\begin{array}{l}1.04 \\
(0.44-2.44)\end{array}$ \\
\hline $\begin{array}{l}\text { Management, } \\
\text { Inflammatory \& } \\
\text { Infectious diseases }\end{array}$ & $5 / 76$ & $\begin{array}{l}6.6 \\
(2.8-14.5)\end{array}$ & $\begin{array}{l}0.64 \\
(0.26-1.59)\end{array}$ \\
\hline $\begin{array}{l}\text { Management, Pediatrics } \\
\text { div. }\end{array}$ & $34 / 277$ & $\begin{array}{l}12.3 \\
(8.9-16.7)\end{array}$ & $\begin{array}{l}1.26 \\
(0.87-1.84)\end{array}$ \\
\hline $\begin{array}{l}\text { Management, Gynecology } \\
\text { \& Obstetrics div. }\end{array}$ & $6 / 99$ & $\begin{array}{l}6.1 \\
(2.8-12.6)\end{array}$ & $\begin{array}{l}0.58 \\
(0.25-1.34)\end{array}$ \\
\hline $\begin{array}{l}\text { Management, Cardiology } \\
\text { \& Neurology div. }\end{array}$ & $13 / 70$ & $\begin{array}{l}18.6 \\
(11.2-29.2)\end{array}$ & $\begin{array}{l}2.06 \\
(1.12-3.80)\end{array}$ \\
\hline Management, Cancer div. & $4 / 106$ & $3.8(1.5-9.3)$ & $\begin{array}{l}0.35 \\
(0.13-0.97)\end{array}$ \\
\hline $\begin{array}{l}\text { Management, emergency } \\
\text { care and surgery div. }\end{array}$ & $8 / 100$ & $\begin{array}{l}8.0 \\
(4.1-15.0)\end{array}$ & $\begin{array}{l}0.79 \\
(0.38-1.63)\end{array}$ \\
\hline Support and informatics & $47 / 280$ & $\begin{array}{l}16.8 \\
(12.9-21.6)\end{array}$ & $\begin{array}{l}1.82 \\
(1.31-2.54)\end{array}$ \\
\hline $\begin{array}{r}\text { Innovation and } \\
\text { development }\end{array}$ & $15 / 158$ & $\begin{array}{l}9.5 \\
(5.8-15.1)\end{array}$ & $\begin{array}{l}0.95 \\
(0.55-1.63)\end{array}$ \\
\hline
\end{tabular}

$\S$ Wards that cared for COVID-19 patients. 


\section{Discussion}

\subsection{Statement of the main findings}

We found that HCWs in wards that were caring for COVID-19 patients were at increased risk for infection (adjusted OR 1.95 (95\% CI 1.65-2.32) with the notable exception of HCWs in the infectious diseases and intensive care units (adjusted OR 0.86 (95\% CI 0.66-1.13)), that did not have higher seroprevalences despite being in close contact with many Covid19 patients. We also report that units with similar types of work may vary greatly in seroprevalences and that the profession associated with highest risk was nurse assistant (adjusted OR 1.62 (95\% CI 1.38-1.90)).

\subsection{Strengths and considerations}

Our study is quite large, and we enrolled the majority of the employees ( $>92 \%(12,928 / 15,300)$ ) of the Karolinska University Hospital suggesting that the data are robust. The study exploited the fact that the linkable hospital administrative systems contained pre-collected and exact information on e.g. patient contact, place of work, profession, and the repurposing of hospital units for COVID-19 care. HCWs can, of course, be exposed to SARS-CoV-2 outside the hospital (community transmission). However, the fact that seropositivities clustered in wards re-purposed for COVID-19 care implies that transmission from patients to HCWs must have occurred at least once in such wards. Once introduced, it is of course possible that the HCWs working in the same unit can transmit infections to each other. The fact that some non-COVID-19 wards also had high seroprevalences could be compatible with outbreaks that had been introduced to the units by community transmission to a member of staff, with subsequent spread among the staff in the unit.

The serology test used had higher sensitivity and specificity than most commercial tests, but imperfect performance is of course still a possible problem. Any misclassification would however have resulted in conservative associations.

\subsection{Comparison with others}

Population seroprevalence had not been measured at the start of our study. Indeed, our main idea was to use the hospital staff without patient contact as a proxy for the general population. A small-scale survey by the Swedish National Public Health Agency subsequently reported a very similar seroprevalence (https://www.folkhalsomyndigheten.se/publi cerat-material/publikationsarkiv/p/pavisning-av-antikroppar-efter-gen omgangen-covid-19-i-blodprov-fran-oppenvarden-delrapport-1/). Sever al other reports have found that the population seroprevalences increased gradually over time as the epidemic progressed (Klevebro et al., 2021).

A seroprevalence survey among HCWs $(n=46,117)$ at a New York City-based health system found a $13.7 \%$ seropositivity rate overall (Moscola et al., 2020). Among employees at 22 elderly care centers in Sweden, $23.0 \%(231 / 1005)$ were seropositive and $46.5 \%$ of the seropositive employees did not report symptoms (Lindahl et al., 2020) Another Swedish cohort study of seroprevalence among HCWs at another acute care hospital $(n=2149)$ that was performed during the same time period and used exactly the same serology method found a seropositivity of $19.1 \%$ and an association with patient contact (OR 2.9, 95\% CI 1.9-4.5) (Rudberg et al., 2020). At KUH, the intensive care and infectious disease units had, in general, a high degree of vigilance and used masks as well as long aprons in contact with patients as well as masks in staff meetings. The data indicate a low level of HCW infection at these units suggesting that experience with infectious diseases and the use of PPE among staff might have been effective in preventing disease among HCWs. Less experienced units may not have had similarly vigilant routines in place.

\section{Conclusion}

We find that SARS-CoV-2 transmission was not random, and instead, we found clear clustering of SARS-CoV-2 exposure among HCWs in specific hospital wards. In agreement with previous reports, our results clearly suggest that nosocomial transmission of SARS-CoV-2 is common, in particular among nurse assistants in wards re-purposed to care for COVID-19 patients. Importantly, however, we also show that examples that HCWs in hospital wards prepared for infectious diseases control and caring for high amount of COVID patients did not have increased risk for SARS-CoV-2 exposure. The large variability among units also suggests that with appropriate preventative measures healthcare work-related risks of SARS-CoV-2 infection may be significantly reduced. In severe outbreak situations, HCWs are likely to be more exposed. As the seroprevalences among HCWs in different units varied widely, it is possible that stringent workplace preventive measures may reduce this risk.

\section{CRediT authorship contribution statement}

K. Miriam Elfström: Data curation, Formal analysis, Writing original draft. Jonas Blomqvist: Data curation, Formal analysis, Writing - review \& editing. Peter Nilsson: Funding acquisition, Investigation, Project administration, Resources, Supervision, Writing - review \& editing. Sophia Hober: Investigation, Resources, Writing review \& editing. Elisa Pin: Investigation, Writing - review \& editing. Anna Månberg: Investigation, Writing - review \& editing. Ville N. Pimenoff: . Laila Sara Arroyo Mühr: Investigation, Writing - review \& editing. Kalle Conneryd Lundgren: Conceptualization, Funding acquisition, Resources, Writing - review \& editing. Joakim Dillner: Conceptualization, Funding acquisition, Project administration, Resources, Supervision, Writing - original draft.

\section{Declaration of Competing Interest}

The authors declare that they have no known competing financial interests or personal relationships that could have appeared to influence the work reported in this paper.

\section{Acknowledgments}

Support was obtained from the Karolinska University Hospital, the County Council of Stockholm, Erling-Persson family foundation, KTH Royal Institute of Technology, Creades, and SciLifeLab. The funding agencies have had no role in the design, execution or interpretation of the study or in the decision to submit for publication.

We would like to thank Suyesh Amatya, Helena Andersson, Shaghayegh Bayati, Emine Eken, Pedram Farsi, Yasmin Hussein, Roxana Merino Martinez, Sara Mravinacova, Björn Pfeifer, Ulla Rudsander, Sadaf Sakina Hassan, Ronald Sjöberg, Balazs Szakos, Hanna Tegel, and Emel Yilmaz for excellent technical assistance.

\section{References}

Zhang, Y.-Z., Holmes, E.C., 2020. A Genomic Perspective on the Origin and Emergence of SARS-CoV-2. Cell 181 (2), 223-227.

Bai, Y., Yao, L., Wei, T., Tian, F., Jin, D.-Y., Chen, L., Wang, M., 2020. Presumed Asymptomatic Carrier Transmission of COVID-19. JAMA 323 (14), 1406. https:// doi.org/10.1001/jama.2020.2565.

Cevik, M., Tate, M., Lloyd, O., Maraolo, A.E., Schafers, J., Ho, A., 2021. SARS-CoV-2, SARS-CoV, and MERS-CoV viral load dynamics, duration of viral shedding, and infectiousness: a systematic review and meta-analysis. The Lancet Microbe. 2 (1), e13-e22.

Burki, T., 2020. England and Wales see 20000 excess deaths in care homes. Lancet (London, England). 395 (10237), 1602. https://doi.org/10.1016/S0140-6736(20) 31199-5.

Feaster, M., Goh, Y.-Y., 2020. High Proportion of Asymptomatic SARS-CoV-2 Infections in 9 Long-Term Care Facilities, Pasadena, California, USA, April 2020. Emerg. Infect. Dis. 26 (10), 2416-2419.

Arons, M.M., Hatfield, K.M., Reddy, S.C., Kimball, A., James, A., Jacobs, J.R., Taylor, J., Spicer, K., Bardossy, A.C., Oakley, L.P., Tanwar, S., Dyal, J.W., Harney, J., Chisty, Z., 
Bell, J.M., Methner, M., Paul, P., Carlson, C.M., McLaughlin, H.P., Thornburg, N., Tong, S., Tamin, A., Tao, Y., Uehara, A., Harcourt, J., Clark, S., Brostrom-Smith, C., Page, L.C., Kay, M., Lewis, J., Montgomery, P., Stone, N.D., Clark, T.A., Honein, M. A., Duchin, J.S., Jernigan, J.A., 2020. Presymptomatic SARS-CoV-2 Infections and Transmission in a Skilled Nursing Facility. The New England journal of medicine. 382 (22), 2081-2090.

Meredith, L.W., Hamilton, W.L., Warne, B., Houldcroft, C.J., Hosmillo, M., Jahun, A.S., Curran, M.D., Parmar, S., Caller, L.G., Caddy, S.L., Khokhar, F.A., Yakovleva, A., Hall, G., Feltwell, T., Forrest, S., Sridhar, S., Weekes, M.P., Baker, S., Brown, N., Moore, E., Popay, A., Roddick, I., Reacher, M., Gouliouris, T., Peacock, S.J., Dougan, G., Török, M.E., Goodfellow, I., 2020. Rapid implementation of SARS-CoV-2 sequencing to investigate cases of health-care associated COVID-19: a prospective genomic surveillance study. Lancet. Infect. Dis 20 (11), 1263-1271.

Shah ASV, Wood R, Gribben C, Caldwell D, Bishop J, Weir A, et al. Risk of hospital admission with coronavirus disease 2019 in healthcare workers and their households: nationwide linkage cohort study. BMJ (Clinical research ed). 2020. 371. m3582.

Nguyen, L.H., Drew, D.A., Graham, M.S., Joshi, A.D., Guo, C.G., Ma, W., et al., 2020. Risk of COVID-19 among front-line health-care workers and the general community: a prospective cohort study. The Lancet Public health. 5 (9), e475-e483.

Moscola, J., Sembajwe, G., Jarrett, M., Farber, B., Chang, T., McGinn, T., Davidson, K.W., 2020. Prevalence of SARS-CoV-2 Antibodies in Health Care Personnel in the New York City Area. JAMA 324 (9), 893. https://doi.org/10.1001/jama.2020.14765.

Rudberg, A.-S., Havervall, S., Månberg, A., Jernbom Falk, A., Aguilera, K., Ng, H., Gabrielsson, L., Salomonsson, A.-C., Hanke, L., Murrell, B., McInerney, G., Olofsson, J., Andersson, E., Hellström, C., Bayati, S., Bergström, S., Pin, E., Sjöberg, R., Tegel, H., Hedhammar, M.y., Phillipson, M., Nilsson, P., Hober, S., Thålin, C., 2020. SARS-CoV-2 exposure, symptoms and seroprevalence in healthcare workers in Sweden. Nat. Commun. 11 (1) https://doi.org/10.1038/s41467-02018848-0.

Jespersen S, Mikkelsen S, Greve T, Kaspersen KA, Tolstrup M, Boldsen JK, et al. SARSCoV-2 seroprevalence survey among 17,971 healthcare and administrative personnel at hospitals, pre-hospital services, and specialist practitioners in the Central Denmark Region. Clinical infectious diseases : An official publication of the Infectious Diseases Society of America. 2020.

Garcia-Basteiro, A.L., Moncunill, G., Tortajada, M., Vidal, M., Guinovart, C., Jiménez, A., Santano, R., Sanz, S., Méndez, S., Llupià, A., Aguilar, R., Alonso, S., Barrios, D., Carolis, C., Cisteró, P., Chóliz, E., Cruz, A., Fochs, S., Jairoce, C., Hecht, J., Lamoglia, M., Martínez, M.J., Mitchell, R.A., Ortega, N., Pey, N., Puyol, L., Ribes, M., Rosell, N., Sotomayor, P., Torres, S., Williams, S., Barroso, S., Vilella, A., Muñoz, J., Trilla, A., Varela, P., Mayor, A., Dobaño, C., 2020. Seroprevalence of antibodies against SARS-CoV-2 among health care workers in a large Spanish reference hospital. Nat. Commun. 11 (1) https://doi.org/10.1038/s41467-020-17318-x.

Wrapp, D., Wang, N., Corbett, K.S., Goldsmith, J.A., Hsieh, C.-L., Abiona, O., Graham, B. S., McLellan, J.S., 2020. Cryo-EM structure of the 2019-nCoV spike in the prefusion conformation. Science 367 (6483), 1260-1263.

Neiman, M., Hellström, C., Just, D., Mattsson, C., Fagerberg, L., Schuppe-Koistinen, I., Gummesson, A., Bergström, G., Kallioniemi, O., Achour, A., Sallinen, R., Uhlén, M., Nilsson, P., 2019. Individual and stable autoantibody repertoires in healthy individuals. Autoimmunity. 52 (1), 1-11.

Klevebro S, Bahram F, Elfström KM, Hellberg U, Hober S, Merid SK, et al. Risk of SARSCoV-2 exposure among hospital healthcare workers in relation to patient contact and type of care. medRxiv. 2021.2021.01.28.21250664.

Lindahl, J.F., Hoffman, T., Esmaeilzadeh, M., Olsen, B., Winter, R., Amer, S., Molnár, C., Svalberg, A., Lundkvist, Å., 2020. High seroprevalence of SARS-CoV-2 in elderly care employees in Sweden. Infect. Ecol. Epidemiol. 10 (1), 1789036. https://doi.org/ 10.1080/20008686.2020.1789036. 